\title{
Evaluación en la enseñanza-aprendizaje de un idioma extranjero en la educación remota
}

\author{
Evaluation in the teaching-learning of a foreign language in remote education
}

\author{
HERMIS TOLENTINO QUIÑONES \\ hermis_une@hotmail.com \\ https://orcid.org/0000-0002-9819-1655 \\ Universidad Nacional de Educación "Enrique Guzmán y Valle”
}

\section{RESUMEN:}

Con la llegada del coronavirus 19 (COVID-19), las instituciones educativas optaron por las clases remotas conllevando esto a hacer cambios, ajustes y adaptaciones en la práctica pedagógica; sin embargo, son pocos o nulos los cambios en la modalidad de evaluación, cuando se aprende-enseña un idioma extranjero, razón por la cual, este ensayo tiene como objetivo reflexionar sobre qué y cómo evaluar. Para ello, se presentó la realidad problemática, los antecedentes y las teorías relacionadas a la evaluación y a la educación remota. Se analizaron e interpretaron los datos para llegar a la conclusión que, las actividades de interacción oral con estudiantes de otros países, la producción escrita creativa y colaborativa, el uso de podcast, entre otros, permiten no solo desarrollar las habilidades de comprensión y producción escrita además de la oral, sino que también que, son útiles para llevar a cabo la evaluación sumativa y formativa.

\section{ABSTRACT:}

With the arrival of coronavirus 19 (COVID-19), educational institutions opted for remote classes, leading to changes, adjustments and adaptations in pedagogical practice; However, there are few or no changes in the evaluation modality, when teaching a foreign language is learned, which is why this essay aims to reflect on what and how to evaluate. For this, the problematic reality, the antecedents and the theories related to evaluation and remote education were presented. The data were analyzed and interpreted to reach the conclusion that oral interaction activities with students from other countries, creative and collaborative written production, the use of podcasts, among others, allow not only to develop written comprehension and production skills. In addition to the oral, but also that, they are useful to carry out the summative and formative evaluation.

\section{Palabras Clave:}

Evaluación; lengua extranjera; educación a distancia.

\section{KEYWORDS:}

Evaluation; foreign language; distance education. 


\section{Introducción}

Con la llegada de la emergencia sanitaria, generada por el COVID-19, muchas instituciones educativas han cerrado sus puertas y han pasado de la modalidad presencial a la modalidad remota; y en ese contexto, se hizo (o debió hacer) una implementación de la tecnología en la enseñanza-aprendizaje de idiomas. Esto último, considerando los diversos contextos: disponibilidad tecnológica y conocimientos sobre su uso por parte de docentes y estudiantes, reajustes de los objetivos curriculares y su implementación en la praxis pedagógica (Borromeo, Fernández y Ramírez, 2018). En este contexto (e incluso antes de la pandemia) muchos docentes siguen teniendo un uso limitado al uso de las tecnologías, poca disponibilidad de recursos, falta de capacitación, falta de apoyo de un especialista como un técnico para resolver los problemas del día a día (Gómez, Ramírez, Martínez-González, y Chuc, 2019). Estos son, para llamarlos de alguna forma, macro problemas que a su vez conllevan a los problemas específicos que se presentan en el quehacer diario de los docentes, que van desde la programación hasta la evaluación del proceso enseñanza-aprendizaje.

Además de los problemas de acceso, implementación y uso de la tecnología para llevar a cabo las clases remotas, se encontraron otros inconvenientes relacionados a la praxis pedagógica, porque muchos docentes han creado y adaptado los materiales didácticos a su disposición para poner en práctica el proceso de enseñanzaaprendizaje de los estudiantes (Almodóvar-López, Atiles, Chavarría-Vargas, Dias, y Zúńiga-León, 2020). Se hace necesario precisar que, no siempre con buenos resultados, ya que muchas veces se hace una réplica de las clases presenciales en las clases remotas; prevaleciendo así, las clases magistrales y no dando paso a modelos más activos que implique la colaboración de los estudiantes (Pérez-López, Vázquez Atochero y Cambero, 2020). Esta situación, el creer que las clases presenciales y remotas son iguales, conlleva a pensar que la evaluación también debe hacerse de igual forma, siendo un problema hasta el momento poco considerado por los investigadores educativos pero latente en la práctica didáctica.

El uso de las tecnologías de información y comunicación (TIC) en la enseñanza-aprendizaje de un idioma extranjero se ha incrementado exponencialmente en las clases remotas; sin embargo, los problemas siguen siendo los mismos que se han encontrado anterior a la pandemia. Por ejemplo, el uso del teléfono móvil en la enseñanza de un idioma puede ayudar a estar conectados con los estudiantes, pero la lentitud y, a veces, la falta de conexión hace que se vea afectada la efectividad de este instrumento (Carreras, Gamallo y Díaz, 2018).

Adicional a ello, un contexto de evaluación oral, escrita, entre otros; esto conlleva al estrés del estudiante al elevarse el filtro afectivo y, al mismo tiempo, se crea estrés en el docente. Además, la diversidad de estudiantes: edad, estatus socioeconómico, acceso, uso y conocimiento de los instrumentos tecnológicos, hace que el aprendizaje sea diferente (Fadda, 2019) y, por ende, también debería serlo el modo de la evaluación. Con relación a ello, podemos decir que, al momento de planificar y programar la evaluación, se debe tener en cuenta los diversos factores (internos y externos) porque podrían influenciar en el buen rendimiento de los estudiantes.

En la educación lingüística se desarrollan las habilidades relacionadas a la comprensión y producción oral y escrita a través de diversas actividades (Benucci, 2009); por ejemplo, el Project Word, actividad que transfiere al estudiante la responsabilidad del propio proceso de aprendizaje y desarrolla la competencia socio e intercultural y la competencia relacionada al saber hacer y saber ser (Angius, 2019). Además, el Project Word permite desarrollar la propria autonomía y hacer uso de diversos medios e instrumentos: audio-oral, visual, escrito, multimedial, etc., y al mismo tiempo, permite desarrollar (en primer lugar) las cuatro habilidades básicas: escuchar, leer, hablar y escribir. En ese sentido, otra de las habilidades que se evalúa, pero que poco se desarrolla en clases es la comprensión escrita, es decir, la lectura. Se presume que esta habilidad es poco desarrolla por dos factores: a) una lectura rápida o de saltos, tal como se hace generalmente al usar un teléfono inteligente, o b) la falta de hacer dos o más lecturas (Clemenzi, 2020). En este sentido, se encuentran incongruencias en cuanto a la práctica de la lectura en clases, muchas veces poca o ausente, pero que tiene un peso considerable en la puntuación en la evaluación. 
Si la lectura es trabajada poco en clases, la escritura lo es aún menor; muchas veces, porque se considera que para desarrollar esta habilidad se emplea mucho tiempo, y los docentes dan mayor importancia a la producción oral (el diálogo) (Leyra, 2017). Aunque es necesario referir que, la habilidad de escritura puede permitir reforzar otras habilidades, por ejemplo, se puede desarrollar la comprensión lectora cuando los estudiantes deben realizar resúmenes partiendo de un input escrito. En este sentido, el resumen no solo permite desarrollar la comprensión, sino también la producción textual; sin embargo, es necesario una guía adecuada por parte del docente, de lo contrario, los estudiantes tienden simplemente a eliminar las frases menos relevantes y no llegan a una (re) elaboración completa del texto (Fiorentino, 2020). Entonces, se podría decir que, si en las clases remotas, el docente llegase a trabajar la comprensión y la producción escrita como se debiera, las evaluaciones también podrían hacer uso de estas estrategias y así evitar, por ejemplo, que los estudiantes sean tentados de copiar y hacer uso de traductores.

Evaluar el conocimiento de un idioma a través de la producción escrita, implica no solo llegar al final del curso y pedir redactar un texto, sino que, implica haber desarrollado en clases esta habilidad; porque, por ello, es importante que el docente sepa que muchos estudiantes no tienen conocimiento del proceso de la producción de textos escritos, sobre todo en la planificación y edición, llegando a considerarla como una habilidad aburrida (Leyra, 2017). Por lo general, los estudiantes tienden a unir frases y conceptos poco compactos, desordenados e incluso sin parafrasear las fuentes, y más aún, hay ocasiones donde se llega al copia y pega (Clemenzi, 2020). En ese sentido, el docente debe proporcionar inputs creativos haciendo uso de otros task creativos acompañados de textos, gráficos, imágenes, audios, videos, entre otros.

En cambio, respecto a la producción oral, es una habilidad que muchos docentes la ponen en el primer plano; sin embargo, el uso de las videoconferencias y al no contar con las herramientas y estrategias correctas, se está convirtiendo en un medio para transmitir información de forma unidireccional; es decir, del docente a los estudiantes (Chambi-Mescco, 2020). Dicho de otra forma, el estudiante se vuelve un agente pasivo, receptor de información. Frente a esto y al considerarse las aulas virtuales, como un espacio de interacción entre estudiantes y docentes, es de suma importancia hacer uso del material auténtico para incentivar el desarrollo de las competencias comunicativas y lingüísticas del idioma que se está aprendiendo (Tolentino, 2020). Precisamos que, si en clases el estudiante se entrena hablando de diferentes temas e incentivados por los diversos inputs auténticos, la evaluación también debería darse de la misma forma, por ejemplo, pidiendo a los estudiantes describir y comentar una imagen, un video, una frase, un poster, entre otros.

La problemática de qué y cómo evaluar un idioma extranjero en el contexto de las clases remotas, nace no al culminar el ciclo académico, sino que debe ser planteado desde el diagnóstico y la planificación de la labor educativa. Muchas veces, la evaluación del conocimiento y el uso del idioma extranjero se basa en un test o una prueba escrita al final del curso, evaluando simplemente la parte lingüística: gramática y léxico, usando, además, instrumentos válidos para las clases presenciales, donde la presencia del docente hacía que los estudiantes no hicieran uso de traductores y/o diccionarios. Las formas de enseñar han variado, los instrumentos que se usan en las clases remotas son diferentes, entonces, ¿por qué las formas de evaluaciones no han cambiado?

En párrafos anteriores, se ha tratado de poner en evidencia la problemática existente sobre la evaluación en el proceso de enseñanza aprendizaje de un idioma extranjero, notándose que poco o nada se ha cambiado en el contexto de las clases remotas y que, además, no hay congruencia entre lo que se hace en clases con el modo de evaluación y los instrumentos. En ese sentido, en las siguientes líneas de este ensayo se presentarán algunas experiencias didácticas que pueden ayudar a la reflexión sobre cuándo, cómo y qué evaluar.

Se ha demostrado que el aprendizaje cooperativo y el uso de las TIC pueden permitir un mejoramiento en el aprendizaje de un idioma extranjero, en todas las habilidades básicas: escuchar, leer, escribir y hablar; y también, las competencias referidas a la gramática y el léxico (Torres-Cajas y Yépez-Oviedo, 2018). De igual manera, la clase invertida junto al uso de las TIC en las clases remotas (web 2.0, videos, multimedia y las aplicaciones), mejoran el aprendizaje de la competencia gramatical del idioma inglés (Martínez, 2019). 
Entonces, si en clases se enseña a trabajar de forma cooperativa, ¿no sería posible evaluarlos de igual forma? ¿No funciona así la sociedad? ¿No se organiza el trabajo e interactúa de forma cooperativa para conseguir objetivos comunes? Bastaría con que el docente, contextualice una situación para que los estudiantes se organicen y mientras lo hacen pueden y deben usare el idioma que aprenden. En ese caso, la evaluación iniciaría desde el momento en que los estudiantes usan el idioma para organizarse hasta el momento de presentar el producto final.

Otra experiencia, muy significativa, se realizó en Italia al organizar una interacción online, donde los estudiantes tenían que interactuar de forma y oral en el idioma que estaban aprendiendo con sus semejantes de otras naciones (Langé Cinganotto y Benedetti, 2020). Esta estrategia también podría servir para evaluar a los estudiantes debido a que sería una prueba real de interacción y no una prueba fingida con los propios compañeros o con el docente. En cambio, en cuanto a la habilidad oral, no de interacción sino de producción a través de un monólogo, hay una experiencia significativa al usar el podcast, donde los estudiantes no solo escucharon, sino que realizaron los propios podcasts; instrumento, que sirvió en primer lugar, para hacer uso del idioma fuera de las horas de clases y reforzar la pronunciación y la entonación del idioma (Gómez y Palma, 2020). Esto también podría servir para hacer las evaluaciones orales, claro está que los criterios y el peso que se da a cada uno de ellos, debe ser diferente y redactados con claridad en la interacción y en el monólogo para que los estudiantes sepan qué se les va a evaluar.

En cambio, respecto a la redacción, la experiencia dicta que se puede solicitar a los estudiantes la producción de un texto escrito, haciendo uso de un input escrito, tal producción escrita puede ser de tres tipos: un comentario, una disertación y un texto creativo (Guerriero, 2019). Es importante subrayar que, este tipo de pruebas tienen que ser adaptadas de acuerdo con el nivel lingüístico y solicitadas en forma de mail, blog, cartas, entrevistas, entre otras. Además, la escritura colaborativa también puede ser una excelente forma de producir (para ser evaluados) a través del Google drive, tal como se hizo en una investigación basada en la hipótesis de la interacción y la teoría sociocultural, donde los estudiantes emplearon sus saberes previos para llegar a conclusiones conjuntas y plasmarlas por escrito (Rosales, Gómez y Carrillo, 2017). A estas experiencias, se suma el uso del Movie Maker, instrumento que sirve para crear historias digitales de forma creativa, ya sea de forma individual o colaborativa, debido a que permite un mejoramiento significativo en el uso del léxico, la gramática y, sobre todo, en la creatividad (Otárola, Díaz, y Cuitińo, 2020).

Sea cual sea el instrumento que se desea utilizar para la evaluación en el proceso de enseńanza-aprendizaje de un idioma extranjero, es necesario considerar el nivel de conocimiento del idioma de los estudiantes, las herramientas con las que se cuenta y especificar los criterios de evaluación; pero, sobre todo, se trata de evaluar tal como se practicó en clases: usando las tecnologías y las diferentes estrategias. En este sentido, es importante conceptualizar dos argumentos generales: la evaluación y la educación remota.

\section{Evaluación}

Es un proceso amplio y al mismo tiempo complejo que usa diversos instrumentos (exámenes, pruebas, observaciones, fichas de cotejo, entre otros) para interpretarlos y dar un juicio de valor relacionadas con la historia personal del estudiante, las condiciones psicológicas y físicas de este, la interacción social, su participación en el proceso de enseńanza-aprendizaje (Berrettini, 2009). Entonces, la evaluación toma en consideración todo el ser del estudiante y los estímulos al cual es expuesto para que, de forma general la institución educativa y de forma específica, el docente, puedan tomar decisiones educativas. En esta línea de pensamiento, se tiene lo que se conoce como la Evaluación para el Aprendizaje (EpA) donde el estudiante es el principal agente de aprendizaje y la interacción con el docente genera una mayor autorregulación y motivación por el aprendizaje y por ende se obtiene mejores resultados educativos (Púñez, 2015); es decir, la evaluación es un proceso continuo donde interactúan los estudiantes y docentes durante todo el proceso de enseñanza-aprendizaje. 
El tipo de evaluación a la cual se hace referencia en este ensayo se apoya en el enfoque socioformativo, vigente sobre todo en la educación remota, debido a que considera que, el trabajo colaborativo y la resolución de problemas en el contexto de la sociedad del conocimiento y el uso de las TIC (Berlanga y Júarez-Hernández, 2020). Requiriendo de esta manera, una evaluación por competencias donde se considere aspectos relacionados, en primer lugar, a los valores y las actitudes de los estudiantes, y en segundo lugar, a los aspectos relacionados con los conocimientos, las habilidades y las capacidades adquiridas (Huerta, 2018).

El tipo de evaluación al cual se hace referencia coincide con los principios del Marco Común Europeo para las lenguas, al considerar al estudiante como un agente social que es parte de una sociedad y que usa el idioma en diversas circunstancias; principalmente, desarrollando la competencia lingüística-comunicativa que implica comprender, expresar e interactuar con otros agentes activos de la sociedad (Consejo de Europa, 2002). Entonces, se podría decir que lo que se busca con este tipo de evaluación en el proceso de enseñanzaaprendizaje de un idioma, es preparar al estudiante para integrarse en la sociedad desde el punto de vista comunicativo.

Existen tres tipos de evaluación: diagnóstica, formativa y sumativa; pero aquí se describirán de forma breve las dos últimas. De acuerdo con Berrettini (2009), la evaluación formativa se desarrolla en todo el proceso de enseñanza-aprendizaje, la cual permite direccionar las diversas etapas de aprendizaje, y tiene como objetivo, el monitoreo de los resultados. En cambio, la evaluación sumativa consiste en verificar y certificar el logro de los objetivos propuestos para pasar a la siguiente etapa (nivel) de estudio.

En cualquier tipo de evaluación es importante el proceso de retroalimentación, considerado esto como el soporte que permite revisar el trabajo realizado en todo el proceso de enseńanza-aprendizaje para, de este modo, orientar el aprendizaje de los estudiantes y, al mismo tiempo, motiva la toma de decisiones pedagógicas y educativas de los docentes (Canabal y Margalet, 2017). Además, es importante subrayar sobre la importancia de la autoevaluación y la coevaluación porque permiten a los estudiantes ser partícipes en su formación, en reconocer sus fortalezas y sus debilidades para tomar decisiones y direccionar los propios aprendizajes (Ponce-Aguilar y Marcillo-García, 2020). Entonces, la evaluación formativa y sumativa permiten no solo al estudiante saber sobre cómo se está aprendiendo, sino que también dan información al docente sobre la efectividad de su práctica pedagógica en general.

\section{La educación remota}

Es la educación que se da en su totalidad en la modalidad online, haciendo uso de dispositivos digitales (teléfonos inteligentes, computadoras, tabletas, etc.) y otros medios de comunicación (televisión, radio, etc.), se caracteriza además por contar muchas veces con plataformas y/o aulas virtuales (Moreno y Aziz, 2019). Las virtudes de esta modalidad de enseñanza-aprendizaje se pueden dividir en ventajas pedagógicas y ventajas prácticas, y de acuerdo con Moreno y Aziz (2019), estas son pueden ser: a) mayor acceso, ya que un estudiante puede acceder a las clases desde cualquier parte del mundo y desde cualquier dispositivo (teléfono inteligente, tableta, computadora, etc.); b) aprendizaje colaborativo, ya que permite interactuar con los diversos compañeros y con el docente de forma oportuna para la resolución de problemas; c) aprendizaje activo, porque se hace uso de los recursos interactivos: juegos, ejercicios de simulación, etc.; d) uso de recursos multimediales, sobre todo con material auténtico ya que permite un mayor contacto con el idioma que se aprende.

Así como existen aspectos positivos, también existen aspectos negativos los cuales son sugeridos por los autores mencionados anteriormente, entre estas desventajas se tiene aquellas relacionadas con la brecha digital: no contar con los instrumentos digitales, ausencia o pésima calidad de internet; poco conocimiento sobre el uso de los medios tecnológicos (estudiantes y docentes) y; actividades poco motivadoras e imitaciones de las clases presenciales trasladadas a la virtualidad. 
Entonces, ¿cómo y qué se debe evaluar en las clases remotas cuando se enseña y/o aprende un idioma extranjero? La respuesta, de hecho, no es fácil, y tampoco existen trabajos y experiencias que puedan ayudar a encontrar una respuesta única; sin embargo, a lo largo de este ensayo, se han mencionado experiencias de cómo se desarrollan las habilidades de comprensión y producción, escrita y oral cuando se aprende un idioma. Se ha mencionado; por ejemplo, los beneficios del trabajo colaborativo y la clase invertida para desarrollar autonomía en los estudiantes, tal como sugiere la evaluación formativa basada en el enfoque socio formativo y la Evaluación para el Aprendizaje que, además, va en concordancia con la filosofía de concebir a las clases remotas como un espacio cuyas ventajas son la participación activa y el uso de los recursos multimediales.

También se han mencionado otras experiencias como el uso del podcast para desarrollar la producción oral; asimismo, se ha hecho referencia a la experiencia de interacción oral y escrita online con estudiantes de otras naciones. En cambio, para desarrollar las habilidades de comprensión y producción escrita se puede hacer uso de inputs escritos, solicitando a los estudiantes hacer resúmenes, dar comentarios y/o inventando historias a través de dispositivos como el Google drive, el Movie Maker, ya sea de forma colaborativa y/o individual. Así mismo, el uso de los materiales auténticos oral y/o multimedial permiten desarrollar la habilidad de la comprensión auditiva de una forma más natural. Estas experiencias, coinciden con lo que se busca en la evaluación, ya sea formativa o sumativa, porque permiten el desarrollo de todas las capacidades lingüísticas, comunicativas y creativas de los estudiantes y en situaciones verosímiles, pero para llegar a evaluar de esta forma, es necesario que, durante el desarrollo de las clases, los estudiantes puedan entrenarse haciendo uso de estos instrumentos y estas estrategias didácticas. Es decir, la evaluación no debe basarse simplemente en evaluaciones gramaticales y lexicales, sino debe implicar las habilidades de comprensión, producción e interacción.

\section{Método}

Para la elaboración del ensayo se realizó, en primer lugar, la planificación, después se pasó a la búsqueda de la literatura haciendo uso de repositorios tales como Scielo, Redalyc, EBSCO, entre otros. Después, se pasó a la búsqueda de la literatura científica, la clasificación de estas y la selección de la información pertinente, para después pasar a la redacción del manuscrito y, en este proceso, se hizo uso del gestor Mendeley. Una vez terminada la redacción se pasó a la revisión de esta, haciendo uso además de un corrector ortográfico presente en Word.

\section{Resultados y conclusiones}

De acuerdo a la exposición, revisión y análisis de los datos se concluye que, en la evaluación en el proceso de enseñanza-aprendizaje de un idioma extranjero en la educación remota, se debe hacer uso de estrategias e instrumentos creativos para evaluar no solo las habilidades básicas (escuchar, leer, hablar y escribir), sino también aquellas habilidades integradas (interactuar, resumir, exponer, entre otros) donde el estudiante hace uso no solo del conocimiento del idioma, sino también de su creatividad y de otros saberes. Además, la evaluación que mayor consideración se debe tener es aquella formativa, incentivando la auto y coevaluación para lograr autonomía en los estudiantes e incentivar la mejora continua. En este tipo de evaluación se puede hacer uso de: la interacción online con estudiantes de otras instituciones; la producción escrita de forma individual o grupal haciendo uso de las diferentes aplicaciones (Movie Maker, documentos Google, entre otros); la comprensión y producción a partir de inputs auténticos; entre otros. Además, se concluye que, en el momento de la evaluación (examen) se deben utilizar las mismas estrategias y los mismos instrumentos que se utilizaron en el desarrollo de las clases.

\section{Referencias}

Almodóvar-López, M., Atiles, J., Chavarría-Vargas, A., Dias, M., y Zúñiga-León, I. (2020). La enseñanza remota no viene sin retos. Revista Electrónica Educare, 24, 1-5. doi: 10.15359/REE.24-S.15

Angius, M. (2019). Il Project Word nell'educazione al patrimonio storico-culturale dei cittadini migranti. Italiano LinguaDue, 11(1), 403-422. doi:10.13130/2037-3597/11859 
Benucci, A. (2009). Costruire un sillabo e i contenuti dell'insegnamento: analisi dei bisogni a curriculi mirati al discente. Siena: Università per Stranieri di Siena.

Berrettini, L. (2009). Modelli operativi per la didattica: l'organizzazione di unità di apprendimento. Perugia: Università per Stranieri di Perugia.

Berlanga, M., y Juárez-Hernández, L. (2020). Paradigmas de evaluación: del tradicional al socioformativo. Diálogos sobre educación. Temas actuales en investigación educativa, 11(21), 1-14. doi: 10.32870/dse.v0i21.646

Borromeo, C., Fernández, J., y Ramírez; A. (2018). La tecnología en la enseñanza de idiomas: evolución a través de los métodos. Reencuentro: Balance y perspectivas de la educación en México http://www.redalyc.org/articulo. oa?id=34065195009

Canabal, C., y Margalef, L. (2017). La retroalimentación: la clave para una educación orientada al aprendizaje. Profesorado, revista de curriculum y formación del profesorado, 21(2), 149-170. https://www.redalyc.org/articulo. oa?id=56752038009

Carreras, G., Gamallo, F., y Díaz, R. (2018). El aprendizaje móvil como herramienta de trabajo en la enseñanza de inglés en la Universidad Médica. Rev haban cienc méd, 17(6), 995-1004. http://www.redalyc.org/articulo. oa?id=180459095014

Chambi-Mescco, E. (2020). La videoconferencia como recurso educativo en los tiempos del COVID-19. Investigación En Educación Médica, 9(36), 108-109. doi: 10.22201/fm.20075057e.2020.36.20267

Clemenzi, L. (2020). Mettiamoci a scrivere: l'esperienza del laboratorio Lisa! Italiano LinguaDue, 12(1), 90-110. doi: $10.13130 / 2037-3597 / 13983$

Consejo de Europa (2002). Marco Común Europeo De Referencia para las lenguas: aprendizaje, enseńanza y evaluación. Centro Virtual Cervantes. http://cvc.cervantes.es/ensenanza/biblioteca_ele/marco/default.htm

Fadda, A. (2019). Scuola digitale: l'ambiente di apprendimento Moodle applicato ad un CPIA. Italiano LinguaDue, 11(1), 375-402. doi:10.13130/2037-3597/11858

Fiorentino, G. (2020). Scrivere come pratica sociale: riassumere per capire e per studiare. Italiano LinguaDue, 12(1), 1-24. doi: 10.13130/2037-3597/13980

Gómez, F., y Palma, E. (2020). El podcast en el desarrollo de las habilidades orales en estudiantes de inglés como lengua extranjera. Educere, la revista venezolana de educación, 24(78), 237-251. https://www.redalyc.org/ jatsRepo/356/35663284005/html/index.html

Gómez, C., Ramírez, J., Martínez-González, O., y Chuc, I. (2019). El uso de las TIC en la Enseñanza del Inglés en las Primarias Públicas. REXE. Revista de Estudios y Experiencias en Educación, 18(36),75-94. doi: 10.21703/ rexe.20191836gomez4

Guerriero, A. (2019). Quadri teorici, pratiche didattiche e tipi di protocolli delle prove di scrittura. Italiano LinguaDue, 11(1), 166-176. doi: 10.13130/2037-3597/11851

Huerta, M. (2018). Evaluación basada en evidencias, un nuevo enfoque de evaluación por competencias. Revista de Investigaciones de La Universidad Le Cordon Bleu, 5(1), 159-171. doi: 10.36955/RIULCB.2018v5n1.0011

Langé, G., Cinganotto, L., y Benedetti, F. (2020). Interazione online: una sperimentazione italiana. Italiano LinguaDue, 12(1), 603-612. doi: 10.13130/2037-3597/13948

Leyra, C. (2017). Producción textual de fábulas en inglés. La colmena, Revista de la Universidad Autónoma del Estado de México, 93(1), 65-79. https://dialnet.unirioja.es/servlet/articulo?codigo=6093587

Martínez, M. (2019). El modelo pedagógico de clase invertida para mejorar el aprendizaje del idioma inglés. Revista Cientifica Investigación Valdizana, 13(4), 204-213. doi: 10.33554/riv.13.4.486

Moreno, A. y Aziz, C. (2019). Formación continua online: Aprendizajes para el futuro de los líderes educativos. Informe Técnico No 7. Líderes educativos. Centro de Liderazgo para la Mejora Escolar:. 
Otárola, L., Díaz, C., y Cuitińo J. (2020). Historias digitales mediante Windows Movie Maker, Una herramienta para mejorar la escritura en inglés. Entramado, 16(1), 122-136. https://doi.org/10.18041/1900-3803/entramado.1.6083

Pérez-López, E., Vázquez Atochero, A., y Cambero, S. (2020). Educación a distancia en tiempos de COVID-19: Análisis desde la perspectiva de los estudiantes universitarios. RIED. Revista Iberoamericana de Educación a Distancia, 24(1), 331-350. https://doi.org/10.5944/ried.24.1.27855

Ponce-Aguilar, E., y Marcillo-García, C. (2020). Autoevaluación y coevaluación: una experiencia en el proceso de enseñanza-aprendizaje. Dominio de Las Ciencias, 6(2), 246-260. https://doi.org/10.23857/dc.v6i3.1216

Púñez, F. de M. (2015). Evaluación para el aprendizaje: una propuesta para una cultura evaluativa. Horizonte de La Ciencia, 5(8), 87-96. https://doi.org/10.26490/uncp.horizonteciencia.2015.8.124

Rosales, L. U., Gómez, L., y Carrillo, K. (2017). Escritura colaborativa de textos argumentativos en inglés usando Google Drive. Estudios pedagógicos, 43(1), 331-348. https://doi.org/10.4067/S0718-07052017000100019

Tolentino, H. (2020). Uso del material auténtico en las aulas virtuales para el aprendizaje de un idioma extranjero. Revista Educación, 45 (1), 598-606. https://doi.org/10.15517/revedu.v45i1.42297

Torres-Cajas, M. y Yépez-Oviedo, D. (2018). Aprendizaje cooperativo y TIC y su impacto en la adquisición del idioma inglés. Revista mexicana de investigación educativa, 23(78), 861-882. Recuperado, de http://www.scielo.org.mx/ scielo.php?script=sci_arttext\&pid=S1405-66662018000300861\&lng=es\&tlng=es.

\section{Como citar este artículo:}

Tolentino, H. (2021). Evaluación en la enseñanza-aprendizaje de un idioma extranjero en la educación. Revista Peruana de Investigación e Innovación Educativa, 1 (1), 31-43 